\title{
miR-429 Suppresses Proliferation and Migration in Glioblastoma Cells and Induces Cell-cycle Arrest via Modulating Several Target Genes of ERBB Signaling Pathway
}

Fatemeh Gheidari ( $\sim$ fatemehgheidari@ut.ac.ir)

University of Tehran College of Science https://orcid.org/0000-0002-0721-4322

\section{Ehsan Arefian}

University of Tehran College of Science https://orcid.org/0000-0002-0758-4710

Mahboubeh Kabiri

University of Tehran College of Science

Ehsan Seyedjafari

University of Tehran College of Science

Ladan Teimoori-Toolabi

Pasteur Institute of Iran

Masoud Soleimani

Tarbiat Modares University Faculty of Medical Sciences

\section{Research Article}

Keywords: glioblastoma multiforme, proliferation, migration, miR-429, ERBB pathway, EGFR, MYC

Posted Date: February 16th, 2021

DOl: https://doi.org/10.21203/rs.3.rs-186982/v1

License: (c) (i) This work is licensed under a Creative Commons Attribution 4.0 International License.

Read Full License 


\section{Abstract}

Glioblastoma is aggressive and lethal brain cancer, which is incurable by cancer standard treatments. miRNAs have great potential to be used for gene therapy due to their ability to modulate several target genes simultaneously. We found miR-429 is downregulated in glioblastoma and has several predicted target genes from the ERBB signaling pathway using bioinformatics tools. ERBB is the most overactivated genetic pathway in glioblastoma patients, which is responsible for augmented cell proliferation and migration in glioblastoma multiforme (GBM).

Here we overexpressed miR-429 using lentiviral vectors in GBM U-251 cells and observed that the expression level of several oncogenes of the ERBB pathway, EGFR, PIK3CA, PIK3CB, KRAS, and MYC significantly decreased; as shown by real-time PCR and western blotting. Using the luciferase assay, we showed that miR-429 directly targets MYC, BCL2, and EGFR. In comparison to scrambled control, miR-429 had a significant inhibitory effect on cell proliferation and migration as deduced from MTT and scratch wound assays and induced cell-cycle arrest in flow cytometry.

Altogether miR-429 seems to be an efficient suppressor of the ERBB genetic signaling pathway and a potential therapeutic for glioblastoma.

\section{Introduction}

Glioblastoma multiforme (GBM) is the most frequent type of malignant brain and other CNS tumor in adults ( $14.6 \%$ of all tumors and $48.3 \%$ of malignant tumors) ${ }^{1}$. About 3.2 per 100,000 people are diagnosed with glioblastoma multiforme annually with a five-years survival rate of $6.8 \%$ post-prognosis in the case of receiving therapy ${ }^{1}$. Fast growth and high mobility of glial cells ${ }^{2}$, genetic heterogeneity of glioblastoma tumors ${ }^{3}$, presence of stem-like cancer cells ${ }^{4}$, and blood-brain barrier (BBB) that limits the immune system to function in the brain ${ }^{5}$, made glioblastoma highly lethal and incurable with conventional treatments of cancer such as chemotherapy and radiotherapy ${ }^{6}$. Poor prognosis and high recurrence rate of glioblastoma highlight the urgent need for novel therapeutic strategies.

Gene-therapy is promising for cancer treatment ${ }^{7-9}$. Various gene-therapies are in clinical trial phases for glioblastoma, which aims to induce the expression of therapeutic genes, such as tumor suppressor, suicide, and immunostimulatory genes, or suppress the expression of oncogenes ${ }^{10}$. miRNAs are natural 22-24 nucleotides long oligonucleotides, which play a role in genetic network regulation at the translation level by binding to complementary regions of 3'-UTRs of mRNAs and blocking their translation ${ }^{11}$. miRNAs are great candidates in gene therapy owing to their ability to suppress the expression of genes of interest ${ }^{12-14}$. miRNAs expression level changes during different physiological and pathological conditions of the body and directly correlates with changes in their target genes expression profiles $^{15-19}$. We can also change the miRNAs' target genes' level by exogenous expression of miRNAs for therapeutic means ${ }^{20-23}$. 
To date, about 140 genetic mutations have had a role in glioblastoma multiforme progression ${ }^{24}$. GBM patients usually have more than one mutation and sometimes hypermutations (about 60 mutations per tumor $)^{25}$. EGFR (epidermal growth factor receptor), a transmembrane glycoprotein, functioning as a receptor tyrosine kinase in the ERBB signaling pathway, is amplified in up to $60 \%$ of GBM patients showing to have a role in cell proliferation, growth, and survival ${ }^{26}$. The activation of EGFR by binding to its ligand triggers its downstream pathways such as phosphatidylinositol-3-kinase (PI3K)/ Protein Kinase $B(A K T)$ and the mammalian target of rapamycin (mTOR) or RAS/RAF/MAPK (mitogen-activated protein kinases) ${ }^{26}$. Both pathways are also highly activated in GBM and subject to other activating mutations in GBM patients, including PDGFRA (10\%), FGFR (3.2\%), PI3K (25\%), PTEN (41\%), NF1 (10\%), KRAS (1\%) and BRAF $(2 \%)^{27}$. ERBB signaling activation leads to the induction of tumor progression, invasion, angiogenesis, and chemotherapy resistance ${ }^{28}$. The use of miRNAs to target ERBB pathway oncogenes decreased proliferation of GBM cells in vitro 29,30 .

Here we overexpressed miR-429 in the GBM U-251 cell line and studied its effect on direct and indirect regulation of several ERBB signaling pathway oncogenes, cell proliferation, migration, and apoptosis rate.

\section{Materials And Methods}

\subsection{In silico miRNA/target selection}

We used the miRWalk 2.0 online tool ${ }^{31,32}$, the Gene-miRNA-pathway tab (http://zmf.umm.uniheidelberg.de/apps/zmf/mirwalk2/path-self.html) to predict miRNAs which suppress the ERBB signaling pathway. We chose miR-429 as a candidate with several oncogenes from the ERBB pathway predicted to be targeted by that; so we analyzed a miRNA microarray dataset (GSE90603) from the GEO database (Gene Expression Omnibus from NCBI website) ${ }^{33}, 34$ (https://www.ncbi.nlm.nih.gov/gds) to investigate miR-429 expression changes in glioblastoma regarding healthy tissue. Further, to look miR-429 targets in more detail, we got the table of predicted targets of miR-429 from TargetScan online tool ${ }^{35}$ (http://www.targetscan.org/vert_71/) and the components of the ERBB signaling pathway from KEGG website $^{36-38}$ (https://www.genome.jp/kegg/pathway.html) comparing them by using the Venn diagram tool from Bioinformatics \& Evolutionary Genomics website (http://bioinformatics.psb.ugent.be/webtools/Venn/). We investigated the expression level changes of the miR-429 predicted target genes from the ERBB pathway in glioblastoma versus normal brain in the Expression Atlas (from EMBL website) ${ }^{39-41}$ (https://www.ebi.ac.uk/gxa/home) and their alterations (mutations or copy number variations) in the TCGA-GBM project (The Cancer Genome Atlas website) (https://portal.gdc.cancer.gov/projects/TCGA-GBM).

\subsection{MiRNA cloning}

We obtained the sequence of miR-429 stem-loop from the miRBase website ${ }^{42,43}$ (http://www.mirbase.org/). Using a nucleic acid editing tool, from the Genome Data viewer (NCBI website) (https://www.ncbi.nlm.nih.gov/genome/gdv/), 200 nucleotides were added to each side of the stem-loop 
to assure its right folding and make its cloning easier. We designed specific primers and added EcoRI and BamHI restriction enzymes sites to them, after checking that they won't cut the sequence desired for cloning. Forming the right miR-429 stem-loop was seen in the RNAfold WebServer online tool (http://rna.tbi.univie.ac.at/cgi-bin/RNAWebSuite/RNAfold.cgi). We performed the polymerase chain reaction (PCR) and then digested the PCR product by restriction enzymes (Thermo Fisher Scientific, Waltham, MA). They were ligated by T4 DNA Ligase (Thermo Fisher Scientific) to pCDH-GFP-Puro (System Biosciences, Palo Alto, CA) Lentiviral vector.

\subsection{Cell culture}

U-251 typical glioblastoma cell line and Human embryonic kidney cells (HEK293T) were purchased from the Iranian Biological Resource Center (IBRC) and characterized by Short Tandem Repeat (STR) analysis. Cells were cultured in Dulbecco's modified Eagle's medium (DMEM) (Gibco, Grand Island), supplemented with $10 \%$ fetal bovine serum (Gibco, Grand Island) and Penicillin-Streptomycin, Tissue Culture Grade 1X (Sigma, St. Louis, MO), at $37^{\circ} \mathrm{C}$ incubator with $95 \%$ humidity, and $5 \% \mathrm{CO} 2$.

\subsection{Viral packaging and transduction}

We cotransfected the seeded HEK293T cells by lentiviral vector pCDH-GFP-Peuro-miR-429/ scrambled, psPAX2 packaging vector, and pMD2G-VSVG vector with polyethyleneimine (Sigma). For four days, cell supernatants containing secreted recombinant viruses were harvested every 24 hours and stored at $4^{\circ} \mathrm{C}$ adding new media to cells. On the fourth day, cell debris was eliminated from viral supernatants by centrifuge at $2000 \times \mathrm{K} \mathrm{G} / 4^{\circ} \mathrm{C}$ for 10 minutes, followed by filtering with $0.2 \mu \mathrm{m}$ syringe filters. Viral supernatants were aliquoted and stored at $-80^{\circ} \mathrm{C}$ before use. U-251 glioblastoma cells were seeded in appropriate sterile dishes (according to the following cellular assay) at 24 hours before transduction. We used $10 \mu \mathrm{g} / \mathrm{ml}$ polybrene (Sigma) to enhance the transduction of virus-containing supernatants. Fluorescent microscopy assured the efficiency of transduction at 48 hours through GFP detection. We performed the cellular assays in a minimum of $90 \%$ efficiency of transduction. Otherwise, Puromycin 1 $\mu \mathrm{g} / \mathrm{ml}$ (Sigma) was added for 48 hours to enrich and select transduced cells.

\subsection{Gene expression by real-time PCR}

Total RNA with miRNAs were extracted from U-251 cells using TRIzol (Invitrogen) on 72 hours after transduction with miR-429/scrambled viruses and then measured by spectrophotometer (Eppendorf). $5 \mu \mathrm{g}$ of each RNA went through complementary DNA (cDNA) synthesis reactions with random hexamer primer (for total mRNA) or specific stem-loop primers (for miRNAs), by using M-MuLV Reverse Transcriptase enzyme (Thermo Fisher Scientific), based on manufacturer's instruction. Design of RT-stem loop primers,

as well as miRNA forward and reverse primers, was performed based on a previously published method ${ }^{44}$ for miR-429 and SNORD47. Specific primers were designed for evaluation of target genes expression via Real-time PCR, using the Primer-Blast online tool (https://www.ncbi.nlm.nih.gov/tools/primer-blast/). We performed real-time PCR with SYBR Green master mix 2X (Ampliqon, Odense M, Denmark) based on the manufacturer's instruction via ABI 7500 (Applied Biosystems, USA) machine. The quantitative PCR program was 5 minutes of $95^{\circ} \mathrm{C}$ followed by 40 cycles of $95^{\circ} \mathrm{C}$ for 15 seconds and $62^{\circ} \mathrm{C}$ for 1 minute. 2 
$-\triangle \Delta C$ t method was used to calculate expression fold changes of miR-429 and some of its predicted target genes, normalized to $\beta 2 \mathrm{M}$ and SNORD47 as internal controls for mRNAs and miRNAs respectively. PCR reactions were duplicate; we experimented with three biological repeats. Used Primers are in Tables 1 and 2.

Table 1

Specific primers used for quantification of miR-429 by Real-time PCR

\begin{tabular}{|ll|}
\hline SNORD47-RT Primer & GTCGTATGCAGAGCAGGGTATTCGCACTGCATACGACAACCTC \\
\hline SNORD47-F Primer & ATCACTGTAAAACCGTTCCA \\
\hline miR-429-RT Primer & GTCGTATGCAGTGCAGGGTCCGAGGTATTCGCACTGCATACGACACGGT \\
\hline miR-429-F Primer & GGGTGGTAATACTGTCTGGTAA \\
\hline Universal-R Primer & GTGCAGGGTCCGAGGT \\
\hline
\end{tabular}

Table 2

Specific primers used for quantification of target genes by Real-time PCR

\begin{tabular}{|lll|}
\hline & Forward Primer & Reverse Primer \\
\hline B2M & ATGCCTGCCGTGTGAAC & ATCTTCAAACCTCCATGATG \\
\hline PIK3CA & CTCCTCTAAACCCTGCTCATC & CATATCTTGCCGTAAATCATCC \\
\hline PIK3CB & ACTTGGTAATCGGAGGATAGG & GAGTGCTTCAACCTGCTTAG \\
\hline KRAS & CACAGCAGGTCAAGAGGAG & TTATGGCAAATACACAAAGAAAGC \\
\hline EGFR & CGTCCGCAAGTGTAAGAAG & AGGAGTCACCCCTAAATGC \\
\hline BCL2 & GATAACGGAGGCTGGGATG & CAGGAGAAATCAAACAGAGGC \\
\hline MYC & AGCGACTCTGAGGAGGAAC & CTGCGTAGTTGTGCTGATG \\
\hline PRKCA & AATGTGACACCTGCGATATG & GATCTGAAAGCCCGTTTGG \\
\hline SHC1 & TGCAAACAGATCATCGCC & GTGGGTTCCTGAGGTATTG \\
\hline estern blotting & \\
\hline
\end{tabular}

For the extraction of transduced U-251 cells' total protein, we used RIPA lysis buffer supplemented with a protease inhibitor cocktail (Merck) then centrifuged the cell lysates at $10000 \mathrm{X} \mathrm{G} / 4^{\circ} \mathrm{C}$ for 15 minutes. Supernatants containing solubilized proteins were collected and measured based on the BCA method (Thermo Fisher Scientific). $40 \mu \mathrm{g}$ of protein samples were subject to SDS-PAGE gel separation at 150 volts (in triplicate) and then transferred onto a PVDF membrane followed by blocking the membrane with skim milk (Merck) solution. Later, we incubated the membrane in primary antibodies solutions including anti-EGFR (1:1000, Novus Biologicals, USA), anti-PIK3CA (1:5000, Abcam, Cambridge, Britain), antiPIK3CB (1:1000, Abcam), anti-KRAS (1:500, Santa Cruz Biotechnology Inc), anti-MYC (1:1000, Abcam) 
and anti- $\beta$-Actin (1:200; Abcam) mouse monoclonal antibodies. We washed the membrane with PBSTween buffer and exposed it to the secondary antibody solution (1:1000, Abcam) conjugated with horseradish peroxidase. We captured a photo after the addition of ECL Western blot analysis substrate (Thermo Fisher Scientific) to the membrane in the dark, and the density of bands was investigated using GelAnalyzer software 2010a and normalized to ACTB as an internal control.

\subsection{Dual-luciferase reporter assay}

We designed specific primers containing restriction enzyme sites at their $5^{\prime}$ to amplify mRNA $3^{\prime}$ untranslated regions ( $3^{\prime}-$ UTRs) of miR-429 predicted target genes by PCR. After cleavage by Xhol and Notl restriction enzymes (Thermo Fisher Scientific), we inserted 3'-UTRs into psiCHECK-2, a dual-luciferase reporter vector (Promega, Wisconsin) by T4 DNA Ligase. 3'-UTRs/ control plasmids plus miR429/scrambled plasmids were cotransfected to seeded Hek293T cells in 96-well plate by PEI MAX reagent (Sigma) in triplicate. We harvested transfected cells on 48 hours and measured luciferase activity using the Dual-Luciferase Reporter Assay System kit (Promega). Renilla luciferase activity normalized against Firefly luciferase activity.

\subsection{MTT cell proliferation assay}

We transduced U-251 cells plated in a 96-well plate $\left(8 \times 10^{3}\right.$ cells/well) with miR-429/scrambled viruses (4 replicates for each). At 72 hours after transduction, we changed the cells' media with fresh media containing 3-(4, 5-dimethylthiazol-2-yl)-2,5-diphenyltetrazolium bromide (MTT; Sigma) reagent $5 \mathrm{mg} / \mathrm{ml}$, and cells were cultured for another 3 hours at $37^{\circ} \mathrm{C}$ incubator. Then, we removed the media and dissolved violet crystals formed on the surface of the plate in dimethyl sulfoxide via shaking. By a plate reader (BioTek, Winooski), we measured the absorbance at $590 \mathrm{~nm}$ and calculated Viability\% as the relative absorbance of miR-429 treated cells to scrambled treated cells. We experimented with three biological repeats.

\subsection{Scratch wound assay (cell migration assay)}

U-251 cells were cultured and transduced in a 12-well plate $\left(8 \times 10^{4}\right.$ cells/well $)$ with miR-429/scrambled viruses ( 2 replicates for each). At 48 hours after transduction, a cross was scratched in the middle of each well using a sterile tip, make it possible to capture the same visual field at each time point. We washed the cell surface with PBS then added new media. By a digital camera connected to a phasecontrast light microscope, we captured images on the distance between cells at $0 \mathrm{~h}, 24 \mathrm{~h}, 48 \mathrm{~h}$, and $72 \mathrm{~h}$ time points. Pictures were analyzed using Image $\mathrm{J}$ software $1.52 \mathrm{a}$, followed by calculating closure\% as an indicator of cell migration. We experimented with three biological repeats.

\subsection{Annexin $\mathrm{V}$ apoptosis assay}

U-251 cells were seeded and transduced in a 24-well plate $\left(4 \times 10^{4}\right.$ cells/well) with miR-429/scrambled viruses ( 2 replicates for each). At 72 hours after transduction, the cells and their culture media that probably contained apoptotic bodies were collected, centrifuged, and washed once with PBS and once with $1 x$ binding buffer from AnnexinV-PE/7AAD apoptosis detection kit (BD Biosciences). Pellets were 
dissolved in $200 \mu \mathrm{l} 1 \mathrm{x}$ binding buffer and stained by adding five $\mu \mathrm{l}$ AnnexinV-PE and 15 minutes' incubation in the dark. Then cells were washed another washing step by $1 \mathrm{x}$ binding buffer, stained by adding five $\mu \mathrm{I}$ 7AAD, and right after were subject to flow cytometry. Obtained data were analyzed using FlowJo 7.6.1 software. We experimented with three biological repeats.

\subsection{Cell-cycle analysis assay}

U-251 cells were seeded and transduced in a 24 -well plate $\left(4 \times 10^{4}\right.$ cells/well) with miR-429/ scrambled viruses ( 2 replicates for each). At 72 hours after transduction, we harvested the cells by trypsinization. Then washed them with PBS, gently add them to another microtube containing cold $70 \%$ Ethanol on vortex. Fixed cells were kept in the fridge for at least 4 hours before cell-cycle assay. Then we centrifuged the cells, removed Ethanol, and washed the cell pellet once with PBS. We stained cells by adding $200 \mu \mathrm{l}$ of Propidium lodide (Pl; $50 \mu \mathrm{g} / \mathrm{mL}$ ) (Sigma), RNase (1.0 mg/mL) (Thermo Fisher Scientific), and Tryton X100 (Sigma) followed by 40 minutes' incubation at $37^{\circ} \mathrm{C}$ in the dark. Then we performed Flow cytometry (BD Biosciences) on cells and used FlowJo 7.6.1 software for the cell-cycle analysis of the data. We experimented with three biological repeats.

\subsection{Statistical analysis}

Biological repeats of the experiments were statistically analyzed using GraphPad Prism 7.04 (San Diego, $\mathrm{CA}$ ) software. The significancy of the data between miR-429-treated and scrambled-treated groups was investigated by applying Student's t-test. We presented the data as mean \pm standard deviation. $P<0.05$ was considered a statistically significant change.

\section{Results}

\section{1. miR-429 is downregulated while its predicted target genes from the ERBB pathway are upregulated in glioblastoma tissue samples in silico}

First, we investigated the expression changes of miR-429 in glioblastoma patients' tumors versus normal tissues based on the GSE90603 miRNA microarray dataset obtained from the GEO database. miR-429 expression data of 16 tumor tissue and four healthy tissue samples from GBM patients showed significant downregulation of miR-429 in glioblastoma (Fig. 1.A). The differential expression of miR-429 in glioblastoma tumors is $0.67 \pm 0.04$ versus $0.88 \pm 0.11$ in normal tissues $\left({ }^{\star} \mathrm{P}<0.05\right)$.

Next, we predicted targets of miR-429 in the ERBB pathway using the miRWalk, and TargetScan on-line tools. Predicted targets of miR-429 in TargetScan/ miRWalk and their commonality with ERBB signaling pathway genes (from KEGG database) are shown in the Venn diagram using Bioinformatics \& Evolutionary Genomics website (Fig. 1.B, Table 3). Based on TargetScan, 15 target genes, and miRWalk, six target genes of miR-429 are members of the ERBB signaling pathway; Three target genes are in both datasets. The miR-429 predicted target genes in the ERBB pathway are upregulated based on the PanCancer analysis of the whole genome-brain on the Expression Atlas database (Fig. 1.C). According to the 
TCGA-GBM project in the TCGA website (Fig. 1.D), these genes are subject to alterations (mutations or copy number variations) in glioblastoma cases. These findings suggest that miR-429 predicted target genes have a role in GBM progression.

Table 3 Predicted targets of miR- 429 which are a member of the ERBB pathway as well, by TargetScan and miRWalk websites

\begin{tabular}{|c|c|c|}
\hline Gene Names & TargetScan & miRWalk \\
\hline ERBB4 & $\mathrm{P}$ & \\
\hline SHC1 & $\mathrm{P}$ & \\
\hline PAK6 & $\mathrm{P}$ & \\
\hline PIK3CB & $\mathrm{P}$ & \\
\hline KRAS & $\mathrm{P}$ & \\
\hline PAK3 & $\mathrm{P}$ & \\
\hline PAK7 & $\mathrm{P}$ & \\
\hline CBL & $\mathrm{P}$ & \\
\hline JUN & $\mathrm{P}$ & \\
\hline NRG1 & $\mathrm{P}$ & \\
\hline GAB1 & $\mathrm{P}$ & \\
\hline PRKCA & $\mathrm{P}$ & \\
\hline PLCG1 & $\mathrm{P}$ & $\mathrm{P}$ \\
\hline CDKN1B & $\mathrm{P}$ & $\mathrm{P}$ \\
\hline PIK3CA & $\mathrm{P}$ & $\mathrm{P}$ \\
\hline EGF & & $\mathrm{P}$ \\
\hline MYC & & $\mathrm{P}$ \\
\hline EGFR & & $\mathrm{P}$ \\
\hline
\end{tabular}

\section{2. miR-429 modulates several ERBB target genes}

To verify the influence of miR-429 on its predicted target genes from the ERBB pathway, we overexpressed miR-429/ scrambled in the U-251 glioblastoma cell line using lentiviral transduction. Overexpression of miR-429 was confirmed by detecting GFP by fluorescent microscopy in 48 hours (Fig. 2.A) and by real-time PCR in comparison to scrambled in 72 hours after transduction. miR-429 was $28.81 \pm 3.13$ fold overexpressed ( $* * P<0.01)$ after transduction with miR-429 Lentivirus compared to scrambled control (Fig. 2.B).

Then we extracted RNA and protein at 72 hours after transduction. Following cDNA synthesis, expression fold changes of mRNAs of several predicted target genes were investigated by real-time PCR with specific primers normalized to $\beta 2 \mathrm{M}$ internal control. The results from biological repeats showed that $P I K 3 C A$ ( ${ }^{*} \mathrm{P}<$ $0.05), P I K 3 C B\left({ }^{*} \mathrm{P}<0.05\right), K R A S\left({ }^{*} \mathrm{P}<0.05\right), E G F R\left({ }^{*} \mathrm{P}<0.05\right), B C L 2(* * \mathrm{P}<0.01), M Y C\left({ }^{*} \mathrm{P}<0.01\right), P R K C A$ $\left({ }^{*} \mathrm{P}<0.05\right)$, and $S H C 1$ ( $\left.{ }^{*} \mathrm{P}<0.05\right)$ mRNA expression levels significantly decreased following miR-429 overexpression (Fig. 2.C).

The protein expression level of five predicted target genes was also investigated by western-blotting normalized to $\beta$-Actin internal control (ACTB). All five selected target genes, EGFR $\left({ }^{*} \mathrm{P}<0.05\right), P I K 3 C A\left({ }^{*} \mathrm{P}\right.$ $<0.05), P I K 3 C B(* * \mathrm{P}<0.01), K R A S\left({ }^{*} \mathrm{P}<0.01\right)$, and $M Y C\left({ }^{*} \mathrm{P}<0.05\right)$, had a significant protein level 
decreased after overexpression of miR-429 in comparison to scrambled (Fig. 2.D, 2.E). Altogether, we can say that miR-429 modulates several target genes (known as oncogenes) from the ERBB signaling pathway.

\section{3. miR-429 directly targets MYC, BCL2, and EGFR}

To verify the direct target genes of miR-429, we cloned 3'-UTRs of three target genes, MYC, BCL2, and EGFR in the psiCHECK2.0 vector, downstream of the Renilla luciferase coding sequence. Then, we cotransfected miR-429/ scrambled and 3'-UTR vectors to HEK293 cells. At 48 hours, we performed the dualluciferase reporter assay measuring the luciferase activity. The relative luciferase activity was decreased significantly $(* P<0.05)$ in all three cases (Fig. 3.A, 3.B). This result approves direct targeting of these three genes, i.e., MYC, BCL2, and EGFR by miR-429.

\section{4. miR-429 overexpression leads to proliferation and migration suppression in U-251 cells}

To investigate the biological function of miR-429, we performed MTT proliferation assay and scratch assay after overexpression of miR-429 in U-251 glioblastoma cells. MTT assay at 72 hours after transduction showed a significant decrease in viable cells, suggesting that cells had a diminished proliferation rate. The viability of miR-429 transduced cells was $85.87 \% \pm 6.32(* \star P<0.01)$ related to the control group (Fig. 4.A).

In another plate, we made a scratch at 48 hours after transduction, and the closure rate of cells was measured $0,24,48$, and 72 hours after scratch, as an indicator of migration potential. After 72 hours' closure rate was significantly decreased $(* \mathrm{P}<0.05)$ in miR-429 transduced cells in comparison to control (Fig. 4.B, 4.C). So, our results from biological assays suggest miR-429 potential role to inhibit glioblastoma cell proliferation and invasion in vitro.

\section{5. miR-429 overexpression does not cause apoptosis in U- 251 cells, but cell-cycle arrest}

To investigate the miR-429 effect on glioblastoma cells apoptosis, we performed Annexin-PE/7AAD assay 72 hours after transduction. Despite the decrease in cell proliferation and migration, miR-429 did not seem to cause any significant effect on apoptosis (early and late) during 72 hours after transduction, as measured by flow cytometry (Fig. 5.A, 5.B, 5.C).

Further, cell-cycle analysis of miR-429/scrambled transduced U-251 cells was performed at 72 hours to investigate whether a cell-cycle arrest had occurred. After miR-429 transduction, there are significantly fewer cells in the S phase, more in $\mathrm{G} 0+\mathrm{G} 1$ and $\mathrm{G} 2\left({ }^{*} \mathrm{P}<0.05\right)$, miR-429 could induce cell-cycle arrest in U251 glioblastoma cells (Fig. 5.D, 5.E, 5.F).

\section{Discussion}


In recent years, miRNAs have attracted scientists' attention because of their capacity to regulate target genes involved in cancer development and progression. Therefore, their implementation as a tool to suppress cancer cell proliferation and invasion either by induction of apoptosis or cell cycle arrest is raised $^{13,45,46}$. The promising outcome of clinical trials in the application of miRNAs in cancer treatment like anti-miR-122, Miravirsen, has shown the great potentials of miRNAs as therapeutic tools ${ }^{12}$. Herein we showed that the Lentiviral delivery of miRNAs to glioblastoma cells is very efficient. Stable transduction using Lentiviral delivery provides permanent miRNA gene expression via antibiotic selection methods.

U-251 glioblastoma cell line used in this study is a typical model of glioblastoma tumor cells that carries a wide range of genetic mutations. U-251 is mutant for TP53 (encoding P53, a molecular inducer of apoptosis), CDKN2A (encoding P16 and P14, molecular inducers of cell cycle arrest), PTEN (encoding inhibitor molecule of PI3K), NF1 (encoding inhibitor molecule of RAS), and EGFR (encoding epidermal growth factor receptor $)^{47,48}$. EGFR, RAS, and PI3K are members of the ERBB signaling pathway, which is amongst the most overactivated genetic mechanisms in glioblastoma patients, leading to cell proliferation and invasion ${ }^{49}$. So a miRNA that targets and suppresses the ERBB signaling pathway was predicted to be a potential tumor suppressor miRNA.

Our bioinformatical analysis predicted that miR-429 could target several members of the ERBB pathway. These molecular targets were upregulated in glioblastoma tissues comparing to the normal brain in silico and were also subject to alterations (mutations or copy number variations) in glioblastoma patients. Besides, miR-429 expression was reduced in glioblastoma patients samples. These findings suggested that overexpression of miR-429 in glioblastoma cell lines might modulate some of these oncogenes from the ERBB pathway directly or indirectly, so suppress glioblastoma tumor cells proliferation and migration.

Real-time PCR and western blotting results supported the bioinformatical prediction. PIK3CA, PIK3CB, $K R A S, E G F R, M Y C, B C L 2, P R K C A$, and SHC1 mRNAs significantly decreased in Real-time PCR after miR429 transduction in U-251 cells. Also, significant downregulation of PIK3CA, PIK3CB, KRAS, EGFR, and MYC proteins in western blotting occurred. Other predicted targets were not measured, as these seemed functionally more relevant. Luciferase assay confirmed that EGFR, MYC, and BCL2 are direct targets of miR-429. Other potential targets are not in the luciferase assay. Direct targeting of MYC ${ }^{50-52}$ and BCL2 ${ }^{50 \text {, }}$ 53,54 by miR-429 was in previous reports for other cancers. Our data confirm this in glioblastoma. MYC is a transcription factor for the ERBB pathway that facilitates the expression of many genes functioning in cell cycle promotion and growth and, BCL2 is an anti-apoptotic agent that promotes survival. Besides, We identified EGFR as a novel target of miR-429.

The EGFR expression becomes higher in $60 \%$ of glioblastoma patients. It is a receptor tyrosine kinase and triggers its downstream signal pathways like PI3K/AKT/mTOR and RAS/RAF/MEK ${ }^{55}$. Once EGFR becomes active by its ligand or constitutively activating mutations like EGFRviii ${ }^{56}$, it activates other kinases like $\mathrm{PI} 3 \mathrm{~K}$, which affects many genes regulation through the activation of AKT oncogene. Activation of the PI3K/AKT/mTOR pathway can lead to glioblastoma tumors expansion and invasion to 
other tissues ${ }^{49}$ and may also be a result of mutations in PI3KCA, KRAS, PTEN, or NF1 ${ }^{27}$. KRAS activates PI3KCA while PTEN inhibits PI3KCA to phosphorylate AKT, NF1 inhibits KRAS. KRAS is active in most glioblastomas and necessary for the persistence of glioblastoma tumors in mouse models ${ }^{57}$. It also

triggers the oncogenic pathway of MAPK (RAS/RAF/MEK/ERK) in glioblastoma ${ }^{58}$. So, the downregulation of PIK3CA, PIK3CB, KRAS, EGFR, and MYC proteins by miR-429 in our study, suggests a tumor suppressor effect of miR-429 in GBM cell lines.

Our results for MTT proliferation assay and scratch wound assay of U-251 GBM cells confirmed the tumor suppressor effect of miR-429 in glioblastoma. Although miR-429 suppressed proliferation and migration in U-251 cells, it did not induce apoptosis. So we speculate it may induce differentiation in GBM cells with suppressed proliferation, which is along with our results on the cell-cycle arrest of GBM cells by miR-429 come from flow cytometry analysis of cell-cycle. Our previously published data confirm that the neuronal differentiation genetic markers increase by miR-429 overexpression in GBM cells ${ }^{59}$. Consistent with our results, Chen et al. showed downregulation of miR-429 happens in glioblastoma patients samples. Thay investigated the suppressor effect of miR-429 overexpression on migration and invasion of U87 GBM cells and confirmed BMK1 as miR-429 direct target ${ }^{60}$. Dong et al. also showed downregulation of miR-429 in glioblastoma, and they could induce apoptosis and suppress proliferation and invasion in U251 and U87 GBM cells by miR-429 mimics. They confirmed SOX2 as a miR-429 direct target ${ }^{61}$. Although their finding on apoptosis induction by miR-429 is inconsistent with our result, the whole conclusion on the tumor suppressor role of miR-429 in glioblastoma cells is similar. The disagreeing results may come from different delivery methods of miR-429. However, We did not find any significant increase in the apoptosis rate after miR-429 lentiviral transduction to U-251 cells. Otherwise, we present the miR-429 arresting cell-cycle as a new perspective.

Besides, our approach to using miRNAs to modulate a genetic pathway instead of a unique target gene, especially in genetically heterogeneous cancers like glioblastoma, seems to make the bioinformatical prediction more realistic and efficient in vitro or in vivo. Our findings, especially the miR-429 regulatory effect on the ERBB signaling pathway in glioblastoma, suggests its potential role as a therapeutic agent not only in glioblastoma but also in any other cancer or diseases, in which ERBB signaling pathway over activation is the main factor.

\section{List Of Abbreviations}

GBM, BBB, miRNA, EGFR, ERBB, PI3K, AKT, mTOR, RAS, RAF, MAPK, PDGFRA, FGFR, PTEN, NF1, KRAS, BRAF, GEO, TCGA, GFP, PCR, RNA, CDNA, mRNA, B2M, BCL2, MYC, PRKCA, SHC1, ACTB, 3'-UTR, HEK293, MTT, PE, 7AAD, TP53, CDKN2A, BMK1, SOX2, LnCRNA, XIST, EMBL, NCBI, STR, IBRC, DMEM, FBS, CO2, RIPA, BCA, SDS-PAGE, PVDF, PBS, PEI

\section{Declarations}

The authors declare that there is no conflict of interest. 


\section{Data availability:}

The datasets used and/or analyzed during the current study are available from the corresponding author on reasonable request.

\section{Acknowledgment:}

The authors would like to thank the National Institute for Medical Research Development (NIMAD) for the financial support of this work (grant no. 942974). The authors would like to acknowledge Dr. Fatemeh Jamshidi Adegani for her mentorship role in this project and Dr. Marie Shamseddin for native English review.

\section{Author contribution:}

The study was conceptualized by EA and MS. The methodology was given by EA and FG. Formal analysis and investigation were done by FG and EA. Writing and original draft preparation was done by FG and EA. Writing, review, and editing were done by ES, MK and LTT. Funding acquisition was provided by MS and EA. FG performed experiments.

\section{References}

1. Ostrom QT, Cioffi G, Gittleman H, Patil N, Waite K, Kruchko C et al (2019) CBTRUS Statistical Report: Primary Brain and Other Central Nervous System Tumors Diagnosed in the United States in 20122016. Neuro Oncol 21:v1-v100

2. Louis D (2006) Molecular pathology of malignant gliomas. Ann Rev Pathol Mech Dis 1:97-117

3. Schwartzbaum J, Fisher J, Aldape K, Wrensch M (2006) Epidemiology and molecular pathology of glioma. Nat Clin Pract Neurol 2:494-503

4. Séhédic D, Cikankowitz A, Hindré F, Davodeau F, Garcion E (2015) Feature review nanomedicine to overcome radioresistance in glioblastoma stem-like cells and surviving clones. Trends Pharmacol Sci $36: 236-252$

5. Kim S, Harford J, Pirollo K, Chang E (2015) Effective treatment of glioblastoma requires crossing the blood-brain barrier and targeting tumors including cancer stem cells: the promise of nanomedicine. Biochem Biophys Res Commun 468:485-489

6. Alifieris C, Trafalis D (2015) Glioblastoma multiforme: pathogenesis and treatment. Pharmacol Ther 152:63-82

7. Urbanska K, Sokołowska J, Szmidt M, Sysa P (2014) Glioblastoma multiforme an overview. Contemp Oncol 18:307-312

8. Adamson C, Kanu O, Mehta A, Di C, Lin N, Mattox A et al (2009) Glioblastoma multiforme: a review of where we have been and where we are going. Expert Opin Investig Drugs 18:1061-1083 
9. Anjuma K, Shagufta B, Abbas S, Patel S, Khan I, Ali Shah S et al (2017) Current status and future therapeutic perspectives of glioblastoma multiforme (GBM) therapy: A review. Biomed Pharmacother 92:681-689

10. Kane J, Miska J, Young J, Kanojia D, Kim J, Lesniak M (2015) Sui generis: gene therapy and delivery systems for the treatment of glioblastoma. Neuro Oncol 17(Suppl 2):ii24-ii36

11. Ambros V (2004) The functions of animal microRNAs. Nature 431:350-355

12. Lindow M, Kauppinen S (2012) Discovering the first microRNA-targeted drug. J Cell Biol 199:407412

13. Chakraborty C, Sharma A, Sharma G, Priya Doss C, Lee S (2017) Therapeutic miRNA and siRNA: Moving from Bench to Clinic as Next Generation Medicine. Molecular Therapy: Neucleic Acids 8:132-143

14. Yang G, Yin B (2014) The advance of application for microRNAs in cancer gene therapy. Biomed Pharmacother 68:137-142

15. Calin G, Sevignani C, Dumitru C, Hyslop T, Noch E, Yendamuri S et al (2004) Human microRNA genes are frequently located at fragile sites and genomic regions involved in cancers. Proc Natl Acad Sci USA 101:2999-3004

16. Ciafrè S, Galardi S, Mangiola A, Ferracin M, Liu C, Sabatino G et al (2005) Extensive modulation of a set of microRNAs in primary glioblastoma. Biochem Biophys Res Commun 335:1351-1358

17. Novakova J, Slabya O, Vyzula R, Michalek J (2009) MicroRNA involvement in glioblastoma pathogenesis. Biochem Biophys Res Commun 386:1-5

18. Hua D, Mo F, Ding D, Li L, Han X, Zhao N et al (2012) A Catalogue of Glioblastoma and Brain MicroRNAs Identified by Deep Sequencing. OMICS: a Journal of Integrative Biology 16:690-699

19. Berthois Y, Delfino C, Metellus P, Fina F, Nanni-Metellus I, Al Aswy H et al (2014) Differential expression of miR200a-3p and miR21 in grade II-III and grade IV gliomas. Cancer Biol Ther 15:938950

20. Ghaemi S, Arefian E, Rezazadeh Valojerdi R, Soleimani M, Moradi motlagh A, Jamshidi Adegani F (2020) Inhibiting the expression of anti-apoptotic genes BCL2L1 and MCL1, and apoptosis induction in glioblastoma cells by microRNA-342. Biomed Pharmacother 121:109641

21. Moradi motlagh A, Arefian E, Rezazadeh Valojerdi R, Ghaemi S, Jamshidi Adegani F, Soleimani M (2020) MicroRNA-129 Inhibits Glioma Cell Growth by Targeting CDK4, CDK6, and MDM2. Molecular Therapy - Nucleic Acids 19:759-764

22. Allahverdi A, Arefian E, Soleimani M, Ai J, Nahanmoghaddam N, Yousefi-Ahmadipour A et al. MicroRNA-4731-5p delivered by AD-mesenchymal stem cells induces cell cycle arrest and apoptosis in glioblastoma. Journal of Cellular Physiology 2020

23. Li W, Ma M, Dong L, Wang F, LX C, Li X (2011) MicroRNA-34a targets notch1 and inhibits cell proliferation in glioblastoma multiforme. Cancer Biol Ther 12:477-483 
24. Cai X, Sughrue ME (2018) Glioblastoma new therapeutic strategies to address cellular and genomic complexity. Oncotarget 9:9540-9554

25. Wang J, Cazzato E, Ladewig E, Frattini V, Rosenbloom D, Zairis S et al (2016) Clonal evolution of glioblastoma under therapy. Nat Genet 48:768-776

26. Miranda A, Blanco-Prieto M, Sousaa J, Pais A, Vitorinoa C (2017) Breaching barriers in glioblastoma. Part I: Molecular pathways and novel treatment approaches. Int J Pharm 531:372-388

27. Brennan C, Verhaak R, McKenna A, Campos B, Noushmehr H, Salama S et al (2013) The somatic genomic landscape of glioblastoma. Cell 155:462-477

28. Hynes N, MacDonald G (2009) ErbB receptors and signaling pathways in cancer. Curr Opin Cell Biol 21:177-184

29. Kalhori M, Arefian E, Fallah Atanaki F, Kavousi K, Soleimani M (2020) miR-548x and miR-4698 controlled cell proliferation by affecting the PI3K/AKT signaling pathway in Glioblastoma cell lines. Sci Rep 10:1-12

30. Kalhori M, Irani S, Soleimani M, Arefian E, Kouhkan F (2019) The effect of miR-579 on the PI3K/AKT pathway in human glioblastoma PTEN mutant cell lines. J Cell Biochem 120:16760-16774

31. Dweep H, Sticht C, Pandey P, Gretz N (2011) miRWalk - database: prediction of possible miRNA binding sites by "walking" the genes of 3 genomes. J Biomed Inform 44:839-837

32. Dweep H, Gretz N (2015) miRWalk2.0: a comprehensive atlas of microRNA-target interactions. Nat Methods 12:697-

33. Edgar R, Domrachev M, Lash A (2002) Gene Expression Omnibus: NCBI gene expression and hybridization array data repository. Nucleic Acids Res 30:207-210

34. Barrett T, Wilhite S, Ledoux P, Evangelista C, Kim I, Tomashevsky M et al (2013) NCBI GEO: archive for functional genomics data sets-update. Nucleic Acids Res 41:D991-D995

35. Agarwal V, Bell G, Nam J, Bartel D (2015) Predicting effective microRNA target sites in mammalian mRNAs. eLife 4:e05005

36. Kanehisa M, Goto S (2000) KEGG: Kyoto Encyclopedia of Genes and Genomes. Nucleic Acids Res 28:27-30

37. Kanehisa M, Sato Y, Furumichi M, Morishima K, Tanabe M (2019) New approach for understanding genome variations in KEGG. Nucleic Acids Res 47:D590-D595

38. Kanehisa M (2019) Toward understanding the origin and evolution of cellular organisms. Protein Sci 28:1947-1951

39. Papatheodorou I, Fonseca N, Keays M, Tang Y, Barrera E, Bazant W et al (2017) Petryszak,R; Expression Atlas: gene and protein expression across multiple studies and organisms. Nucleic Acids Res 46:D246-D251

40. Petryszak R, Fonseca N, Füllgrabe A, Huerta L, Keays M, Tang Y et al (2017) The RNASeq-er API-a gateway to systematically updated analysis of public RNA-seq data. Bioinformatics 33:2218-2220 
41. Fonseca N, Marioni J, Brazma A (2014) RNA-Seq Gene Profiling - A Systematic Empirical Comparison. PLoS One 9:e107026

42. Kozomara A, Birgaoanu M, Griffiths-Jones S (2019) miRBase: from microRNA sequences to function. Nucleic Acids Res 47:D155-D162

43. Griffiths-Jones S (2004) The microRNA Registry. Nucleic Acids Res 32:D109-D111

44. Naderi M, Abdul Tehrani H, Soleimani M, Shabani I, Hashemi S. A Home-brew Real-time PCR Assay for Reliable Detection and Quantification of Mature miR-122. Appl Immunohistochem Mol Morphol 2015; 23

45. Calin G, Dumitru C, Shimizu M, Bichi R, Zupo S, Noch E et al (2002) Frequent deletions and downregulation of micro- RNA genes miR15 and miR16 at 13q14 in chronic lymphocytic leukemia. Proc Natl Acad Sci USA 99:15524-15529

46. Hydbring P, Badalian-Very G (2013) Clinical applications of microRNAs. F1000Res 2:136

47. Torsvik A, Stieber D, Enger P, Golebiewska A, Molven A, Svendsen A et al (2014) U-251 revisited: genetic drift and phenotypic consequences of long-term cultures of glioblastoma cells. Cancer Med $3: 812-824$

48. Patil V, Pal J, Somasundaram K (2015) Elucidating the cancer-specific genetic alteration spectrum of glioblastoma derived cell lines from whole exome and RNA sequencing. Oncotarget 6:43452-43471

49. McLendon R, Friedman A, Bigner D, Van Meir EG, Brat DJ, Mastrogianakis M (2008) G, et al. Comprehensive genomic characterization defines human glioblastoma genes and core pathways. Nature 455:1061-1068

50. Zhang C, Chang C, Gao H, Wang Q, Zhang F, Xu C (2018) MiR-429 regulates rat liver regeneration and hepatocyte proliferation by targeting JUN/MYC/BCL2/CCND1 signaling pathway. Cell Signal 50:8089

51. Wang H, Wang W, Zhuang H, Xu M (2018) MiR-429 regulates the proliferation and apoptosis of nephroblastoma cells through targeting c-myc. Eur Rev Med Pharmacol Sci 22:5172-5179

52. Sun T, Wang C, Xing J, Wu D (2011) miR-429 modulates the expression of c-myc in human gastric carcinoma cells. Eur J Cancer 47:2552-2559

53. Zhu P, Zhang J, Zhu J, Shi J, Zhu Q, Gao Y (2015) MiR-429 Induces Gastric Carcinoma Cell Apoptosis Through Bcl-2. Cell Physiol Biochem 37:1572-1580

54. Wang Y, Li M, Zang W, Ma Y, Wang N, Li P et al (2013) MiR-429 up-regulation induces apoptosis and suppresses invasion by targeting Bcl-2 and SP-1 in esophageal carcinoma. Cell Oncol (Dordr) 36:385-394

55. Messaoudi K, Clavreul A, Lagarce F (2015) Toward an effective strategy in glioblastoma treatment. Part I: resistance mechanisms and strategies to overcome resistance of glioblastoma to temozolomide. Drug Discov Today 20:899-905

56. Feng H, Hu B, Vuori K, Sarkaria J, Furnari F, Cavenee W et al (2014) EGFRvIlI stimulates glioma growth and invasion through PKA-dependent serine phosphorylation of Dock180. Oncogene 
33:2504-2512

57. Holmen S, Williams B (2005) Essential role for Ras signaling in glioblastoma maintenance. Cancer Res 65:8250-8255

58. Xu P, Zhang G, Hou S, Sha L (2018) MAPK8 mediates resistance to temozolomide and apoptosis of glioblastoma cells through MAPK signaling pathway. Biomed Pharmacother 106:1419-1427

59. Gheidari F, Arefian E, Jamshidi-Adeghani F, Soleimani M (2016) Differentiation Induction Effect of Mir-429 Over-Expression in U251 Glioma Cell Line. International Clinical Neuroscience Journal 3:201-205

60. Chen W, Zhang B, Guo W, Gao L, Shi L, Li H et al (2015) miR-429 inhibits glioma invasion through BMK1 suppression. J Neurooncol 125:43-54

61. Dong H, Hao X, Cui B, Guo M (2017) MiR-429 suppresses glioblastoma multiforme by targeting SOX2. Cell Biochem Funct 35:260-268

\section{Figures}

A)

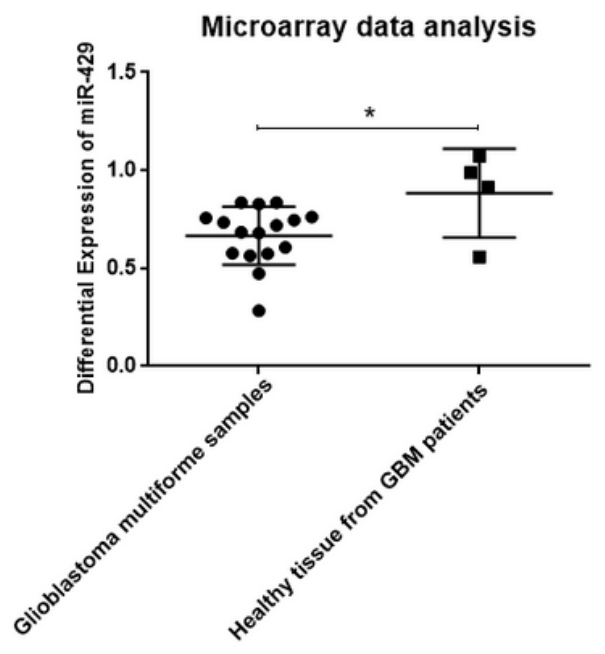

C)

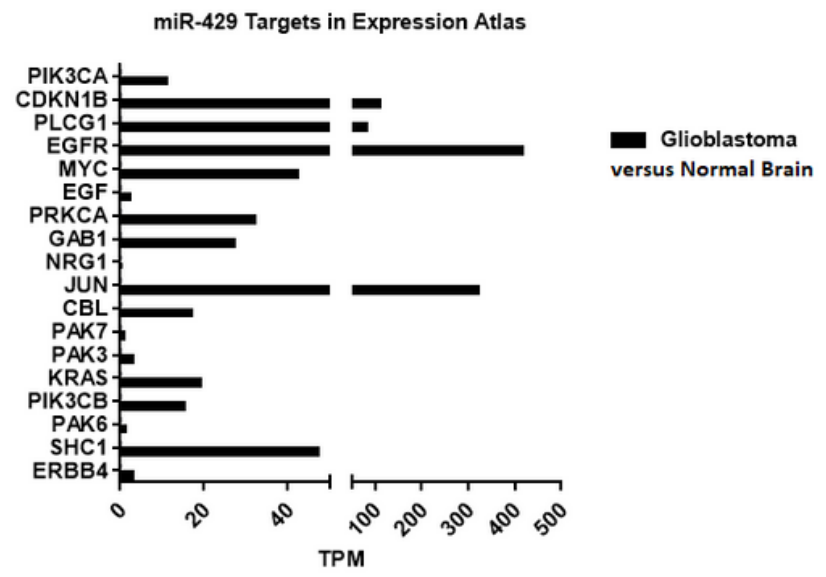

B) ERBB Signaling Pathway Genes Predicted Targets of miR-429 by KEGG

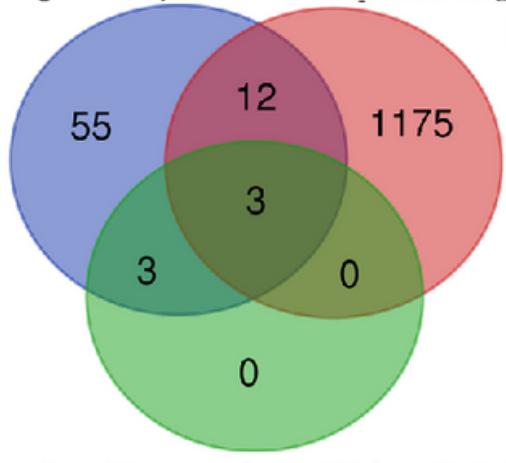

Predicted Targets of miR-429 by miRWalk

D)

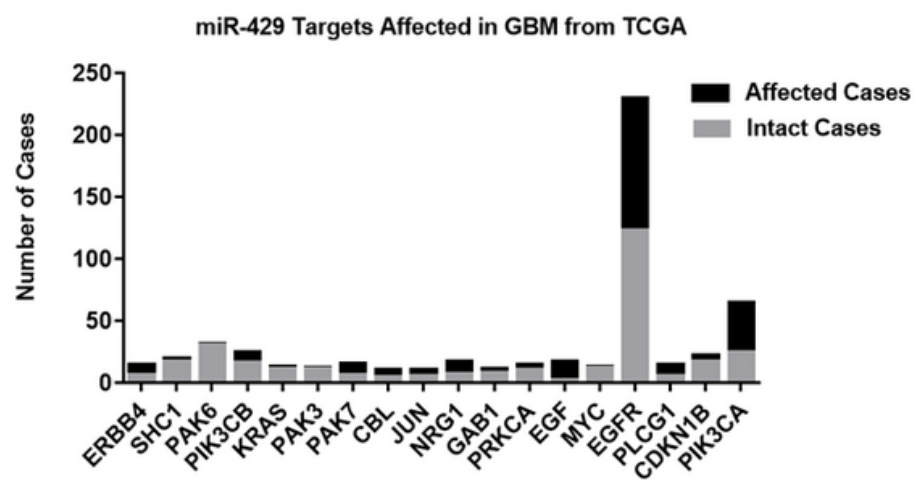


(A) Statistical analysis of a miRNA microarray dataset from GEO (GSE90603) shows significant downregulation of miR-429 in glioblastoma tumor tissues $(n=16)$ versus normal tissues from GBM patients $(n=4)$. Differential expression of miR-429 in glioblastoma tumors is $0.67 \pm 0.04$ versus $0.88 \pm$ 0.11 in normal tissues. Data are shown as mean \pm SD of results from independent biological repeats ( $\left.{ }^{*}<0.05\right)$. GEO, Gene Expression Omnibus; SD, standard deviation. (B) Venn diagram from Bioinformatics \& Evolutionary Genomics website (http://bioinformatics.psb.ugent.be/webtools/Venn/) showing common genes of the ERBB signaling pathway from KEGG website (https://www.genome.jp/kegg/pathway.html) and miR-429 predicted targetome from TargetScan (http://www.targetscan.org/vert_71/) and miRWalk (http://zmf.umm.uniheidelberg.de/apps/zmf/mirwalk2/path-self.html) websites. (C) Overexpression of miR-429 predicted targets from ERBB. The data is drawn from the Expression Atlas (https://www.ebi.ac.uk/gxa/home) website, Pan-Cancer analysis of the whole genome-brain. TPM, transcript per million. (D) Affected cases (mutants or copy number variants) versus intact cases of GBM for miR-429 predicted targets. The data is drawn from the TCGA-GBM project (https://portal.gdc.cancer.gov/projects/TCGA-GBM). TCGA, The Cancer Genome Atlas website.

A)

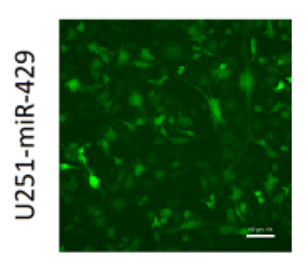

\section{ֻั}

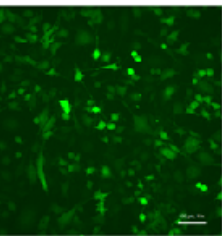

C)

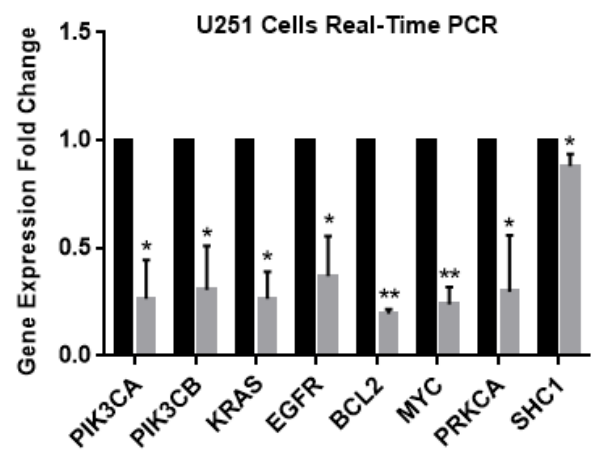

B)

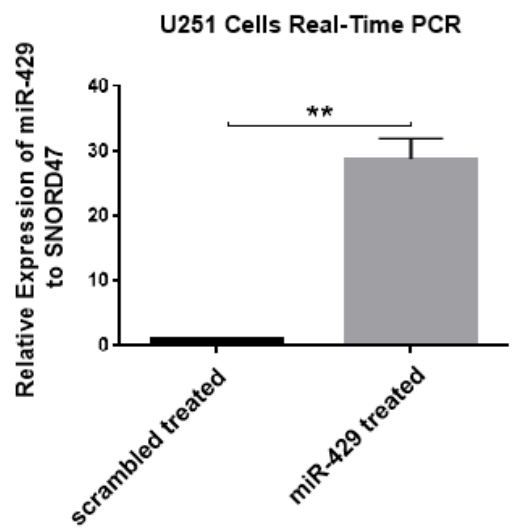

D)

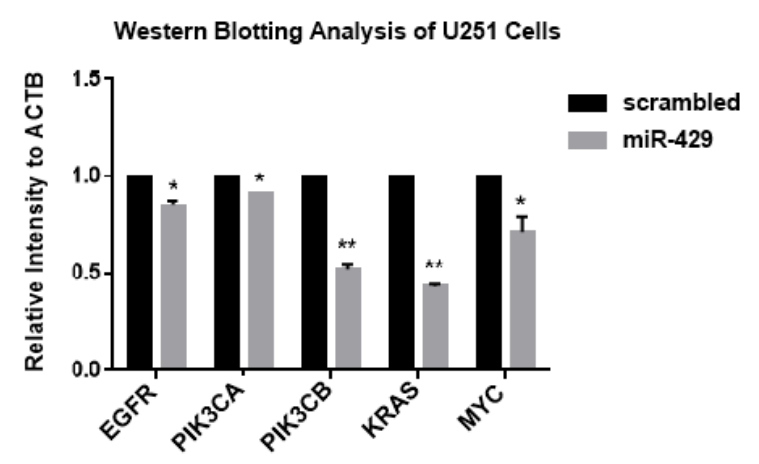

E)

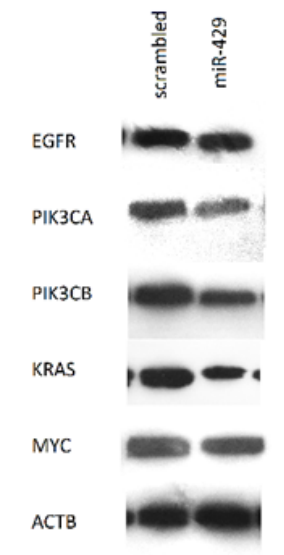

\section{Figure 2}

A) Investigation of transduction efficiency of U-251 cells with miR-429/scrambled Lentiviruses by fluorescent microscopy. (B) miR-429 is significantly over-expressed in U-251 cells, after transduction with miR-429 Lentiviruses. Real-time PCR shows a $28.81 \pm 3.13$ fold overexpression normalized to SNORD47 
internal control. Data are shown as mean \pm SD of results from independent biological repeats $(* \star P<0.01)$. (C) Over-expression of miR-429 significantly alters mRNA levels of predicted target genes, PIK3CA ( $F C=$ $0.27 \pm 0.18), \mathrm{PIK} 3 \mathrm{CB}(\mathrm{FC}=0.31 \pm 0.20), \mathrm{KRAS}(\mathrm{FC}=0.27 \pm 0.13), \mathrm{EGFR}(\mathrm{FC}=0.37 \pm 0.19), \mathrm{BCL} 2(\mathrm{FC}=0.01$ $\pm 0.02), \mathrm{MYC}(\mathrm{FC}=0.24 \pm 0.08), \mathrm{PRKCA}(\mathrm{FC}=0.30 \pm 0.26)$ and $\mathrm{SHC} 1(\mathrm{FC}=0.88 \pm 0.56)$. Gene expression was investigated by qRT-PCR in U-251 glioblastoma cells 72 hours after transduction with miR-429 and scrambled viruses and normalized to $\beta 2 \mathrm{M}$ internal control. Data are shown as mean \pm SD of results from independent biological repeats $\left({ }^{*} P<0.05,{ }^{*} \mathrm{P}<0.01\right)$. qRT-PCR, quantitative real-time polymerase chain reaction; $F C$, fold change; SD, standard deviation. (D) Western-blotting showed significant decrease in the expression of predicted target proteins, EGFR ( $F C=0.85 \pm 0.02)$, PIK3CA $(F C=0.91 \pm 0.00)$, $\mathrm{PIK} 3 \mathrm{CB}(\mathrm{FC}=$ $0.52 \pm 0.02)$, KRAS ( $F C=0.43 \pm 0.01)$, and MYC $(F C=0.71 \pm 0.08)$, normalized to $\beta$-Actin, in U-251 glioblastoma cells 72 hours after transduction with miR-429 and scrambled viruses. Data are shown as mean $\pm S D$ of results from independent biological repeats $\left({ }^{*} P<0.05,{ }^{*} P<0.01\right)$. ACTB, $\beta$-Actin; FC, fold change; SD, standard deviation. (E) Western-blotting showed a significant decrease in the expression of predicted target proteins of miR-429, normalized to $\beta$-Actin, in U-251 glioblastoma cells transduced with miR-429 Lentiviruses.

A)

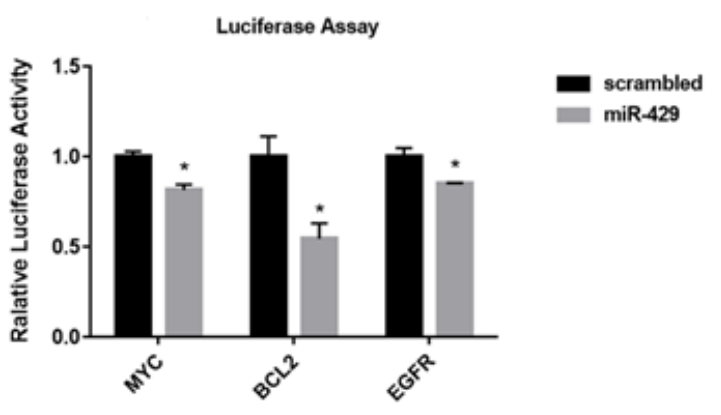

B)

\begin{tabular}{|c|c|c|}
\hline $\begin{array}{l}\text { Position } 2820-2826 \text { of BCL2 3' UTR } \\
\text { hsa-miR-429 }\end{array}$ & $5^{\prime}$ & $\begin{array}{c}\text {...gGCCCCAGAACUGUACAGUAUUG. . . } \\
\qquad\|\|\|\| \|\end{array}$ \\
\hline $\begin{array}{l}\text { Position } 348-354 \text { of MYC } 3 \text { ' UTR } \\
\text { hsa-miR-429 }\end{array}$ & $5^{\prime \prime}$ & $\begin{array}{l}\text {... UAGUAUAUAGUACCU-.-AGUAUUAU... } \\
\qquad||||||||||\end{array}$ \\
\hline $\begin{array}{l}\text { Position } 5849-5855 \text { of EGFR 3' UTR } \\
\text { hsa-miR- } 429\end{array}$ & $5^{\prime \prime}$ & 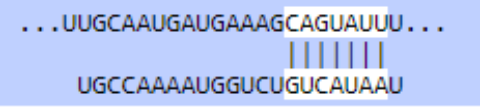 \\
\hline $\begin{array}{l}\text { Position } 5927-5933 \text { of EGFR 3' UTR } \\
\text { hsa-miR-429 }\end{array}$ & $5^{\prime}$ & $\begin{array}{l}\text {...gUUGAUUGUGCAUUGAGUAUUAA... } \\
\qquad \begin{array}{l}\|\| \\
\text { ugCCAAAAUGGUCUGUCAUAAU }\end{array}\end{array}$ \\
\hline
\end{tabular}

\section{Figure 3}

(A) miR-429 directly targets MYC, BCL2, and EGFR mRNAs. Luciferase assay shows a significant decrease in light intensity coming from luciferase enzyme activity cloned at the upstream of MYC $(0.81 \pm$ $0.03), \mathrm{BCL} 2(0.54 \pm 0.08)$ and EGFR $(0.85 \pm 0.00)$ 3'-UTRs in psiCHECK2.0 vector. Data are shown as mean $\pm S D$ of results from independent biological repeats ( $\left.{ }^{*}<<0.05\right)$. UTR, untranslated region. (B) Predicted binding sites of miR-429 on 3'-UTRs of MYC, BCL2, and EGFR mRNAs from TargetScan database. UTR, untranslated region. 
A)

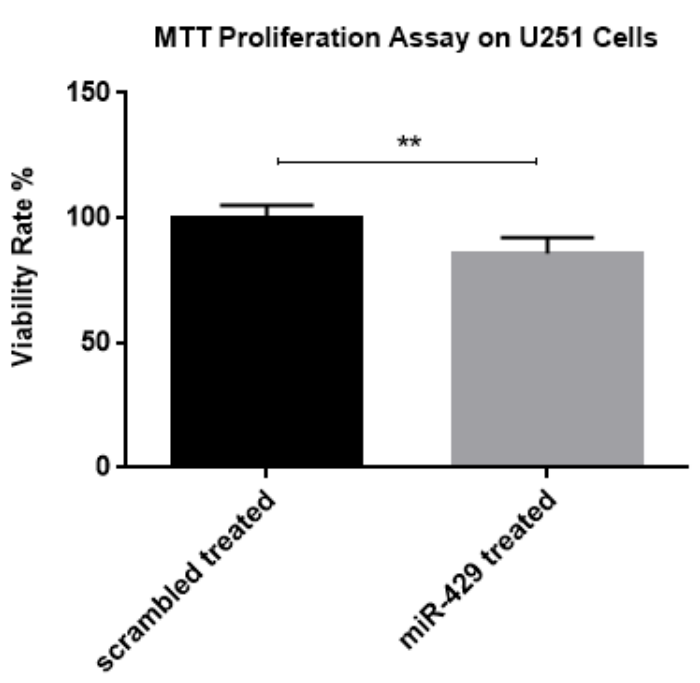

B)

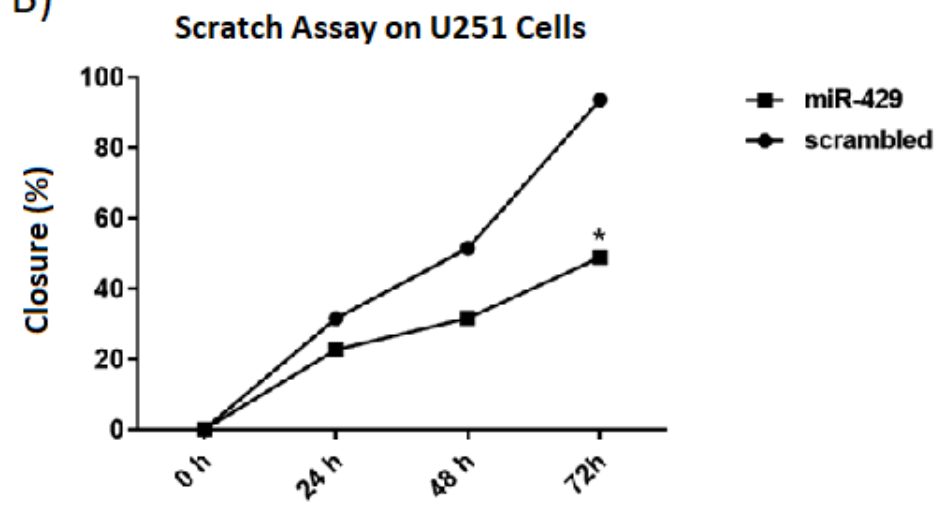

C)

$\mathrm{Oh}$

$24 \mathrm{~h}$

$48 \mathrm{~h}$

$72 \mathrm{~h}$
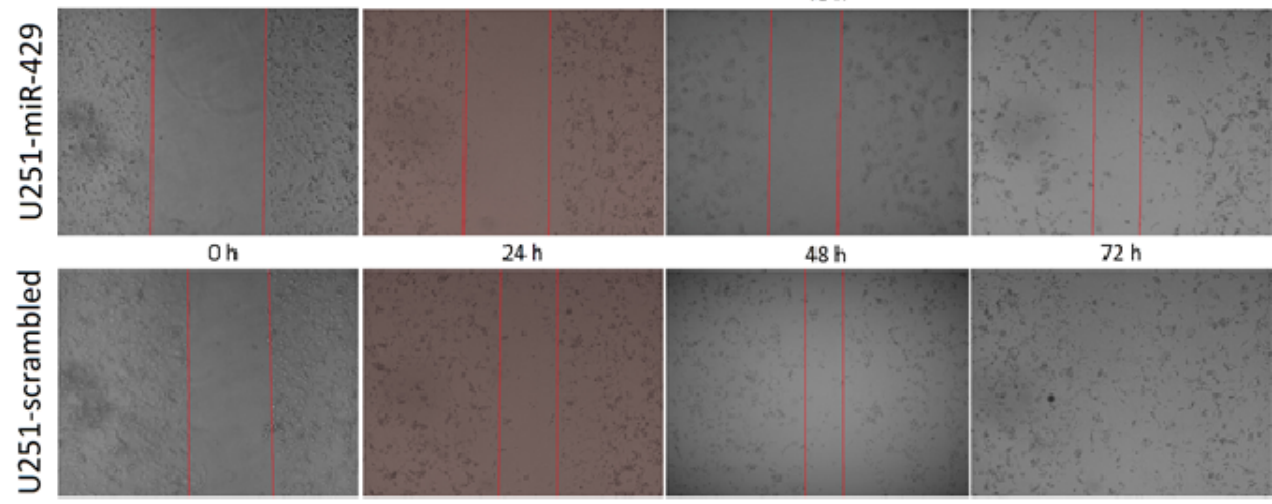

$48 \mathrm{~h}$

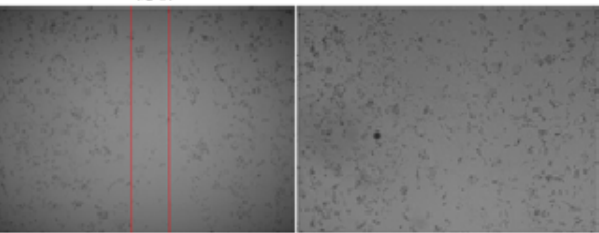

\section{Figure 4}

(A) MTT proliferation assay shows a significant decrease in the viability rate of glioblastoma cells by miR-429 overexpression. The viability rate of U-251 cells transduced with miR-429 viruses is $\% 85.87 \pm$ 6.32 related to scrambled. Data are shown as mean \pm SD of results from independent biological repeats ( $\left.{ }^{\star} \mathrm{P}<0.05\right)$. MTT, 3-(4, 5-dimethylthiazol-2-yl)-2, 5-diphenyltetrazolium bromide. (B) Scratch wound assay shows a significant decrease in the migration rate of U-251 glioblastoma cells, 72 hours after miR-429 overexpression. Closure rate was \% $22.67 \pm 4.94, \% 31.74 \pm 10.01$ and $\% 48.96 \pm 13.70$ for mir-429 transduced cells for 24,48 and 72 hours, respectively and \% $31.61 \pm 1.45, \% 51.54 \pm 3.65$ and \% $93.72 \pm$ 8.88 for scrambled transduced cells, respectively. Data are shown as mean \pm SD of results from independent biological repeats ( $\left.{ }^{*} P<0.05\right)$. (C) Scratch wound assay shows a significant decrease in the migration rate of U-251 glioblastoma cells, 72 hours after miR-429 overexpression. 
A)

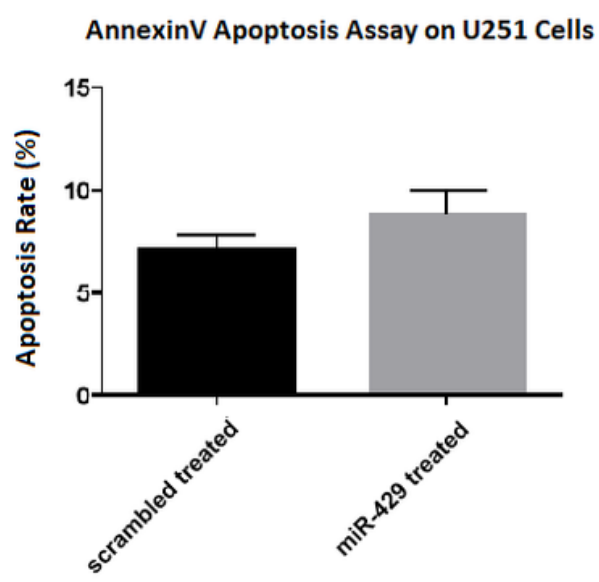

D)

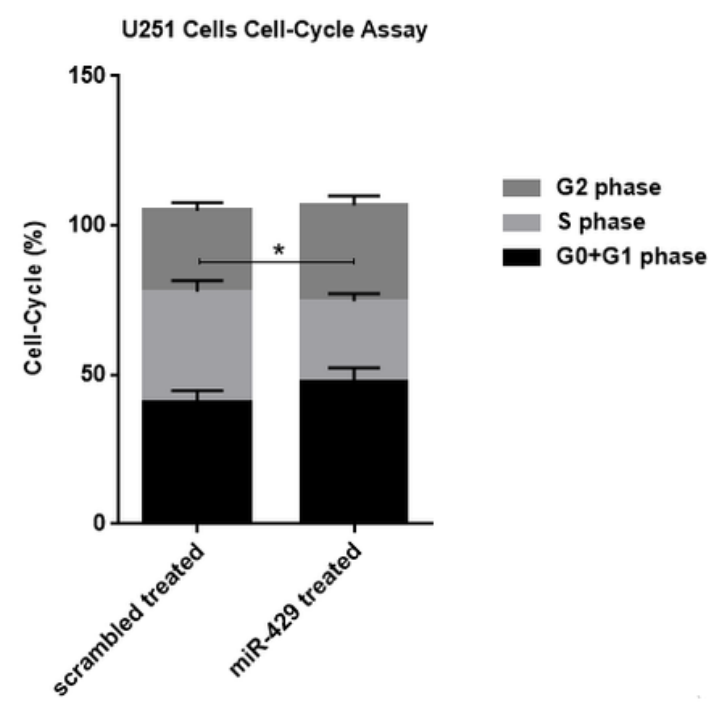

B)

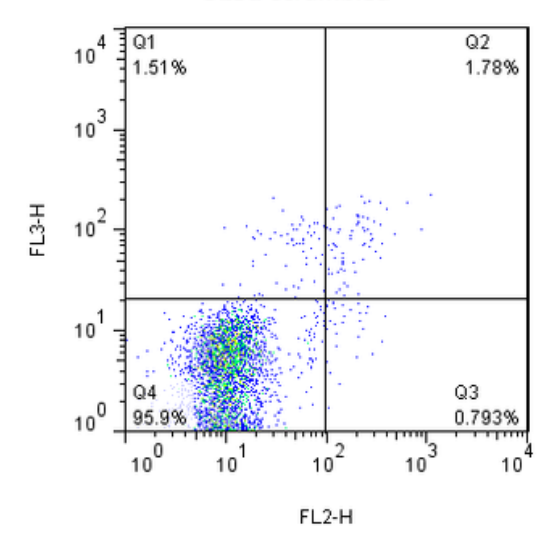

E)

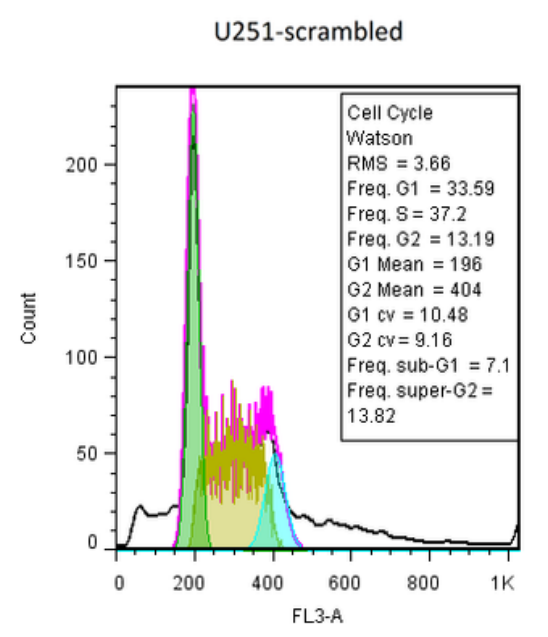

C)

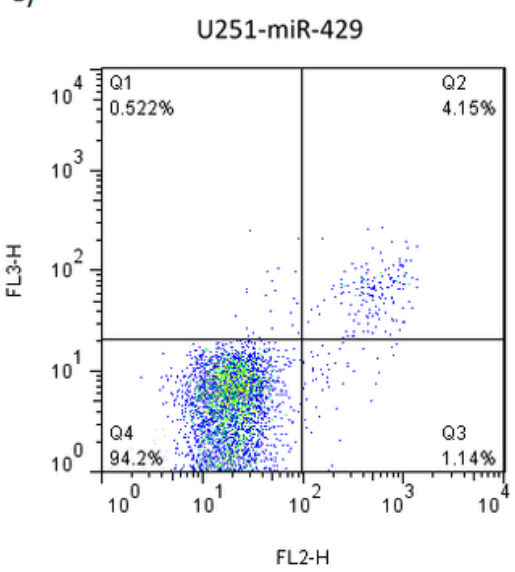

F)

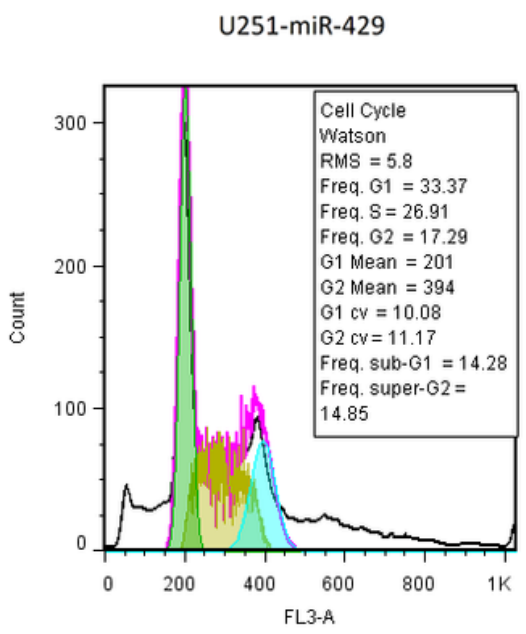

Figure 5

A) AnnexinV apoptosis assay shows that miR-429 doesn't induce significant apoptosis in U-251 glioblastoma cells. Apoptosis rate in flow cytometry of U-251 cells was (B) $\% 7.12 \pm 0.72$ with the scrambled virus and (C) \% $8.87 \pm 1.12$ with the miR-429 virus. (D) Cell-cycle assay shows that miR-429 overexpression significantly reduces cells in the $S$ phase with more cells arrested in the G0+G1 and G2 phases. Flow cytometry results shows (E) \% $40.69 \pm 4$ of cell in G0+G1 phase, \% $37.20 \pm 3.7$ of cell in S phase, and \% $27.01 \pm 2.7$ of cell in G2 phase with the scrambled virus and (F) \% $47.65 \pm 4.8$ of cell in $\mathrm{G} 0+\mathrm{G} 1$ phase, $\% 26.91 \pm 2.7$ of cell in S phase, and \% $32.14 \pm 3.2$ of cell in $\mathrm{G} 2$ phase with the miR-429 virus. Data are shown as mean $\pm \mathrm{SD}$ of results from independent biological repeats $\left({ }^{*}<0.05\right)$.

\section{Supplementary Files}

This is a list of supplementary files associated with this preprint. Click to download. 
- EGFR13.jpg

- KRAS.jpg

- MYC.jpg

- PIK3CA.jpg

- pik3cb.jpg 